University of Wollongong

Research Online

Faculty of Engineering and Information

Faculty of Engineering and Information

Sciences - Papers: Part B

Sciences

2020

Combined General Vector Machine for Single Point Electricity Load Forecast

Binbin Yong

University of Wollongong, yongbb14@Izu.edu.cn

Yongqiang Wei

Jun Shen

University of Wollongong, jshen@uow.edu.au

Fucun Li

University of Wollongong, f1626@uowmail.edu.au

Xuetao Jiang

See next page for additional authors

Follow this and additional works at: https://ro.uow.edu.au/eispapers1

Part of the Engineering Commons, and the Science and Technology Studies Commons

Research Online is the open access institutional repository for the University of Wollongong. For further information contact the UOW Library: research-pubs@uow.edu.au 


\title{
Combined General Vector Machine for Single Point Electricity Load Forecast
}

\author{
Abstract \\ General Vector Machine (GVM) is a newly proposed machine learning model, which is applicable to small \\ samples forecast scenarios. In this paper, the GYM is applied into electricity load forecast based on single \\ point modeling method. Meanwhile, traditional time series forecast models, including back propagation \\ neural network (BPNN), Support Vector Machine (SVM) and Autoregressive Integrated Moving Average \\ Model ( ARIMA), are also experimented for single point electricity load forecast. Further, the combined \\ model based on GYM, BPNN, SVM and ARIMA are proposed and verified. Results show that GYM \\ performs better than these traditional models, and the combined model outperforms any other single \\ models for single point electricity load forecast. \\ Keywords \\ point, vector, combined, single, machine, general, forecast, load, electricity \\ Disciplines \\ Engineering | Science and Technology Studies

\section{Publication Details} \\ Yong, B., Wei, Y., Shen, J., Li, F., Jiang, X. \& Zhou, Q. (2020). Combined General Vector Machine for Single \\ Point Electricity Load Forecast. The 9th International Conference on Frontier Computing (FC2019) Theory, \\ Technologies and Applications (pp. 284-290). LNEE: Springer.

\section{Authors} \\ Binbin Yong, Yongqiang Wei, Jun Shen, Fucun Li, Xuetao Jiang, and Qingguo Zhou
}




\title{
Combined General Vector Machine for Single Point Electricity Load Forecast
}

\author{
Binbin Yong ${ }^{1,2}$, Yongqiang $\mathrm{Wei}^{1}$, Jun Shen ${ }^{3,4},{\text { Fucun } \mathrm{Li}^{3} \text {, Xuetao Jiang }}^{1}$, and \\ Qingguo $\mathrm{Zhou}^{1}$ * \\ 1 School of Information Science and Engineering, \\ Lanzhou University, Lanzhou, Gansu, China \\ \{yongbb, weiyq18, jiangxt 18 , zhouqg\} $@$ lzu.edu.cn \\ 2 School of Physical Science and Technology, \\ Lanzhou University, Lanzhou, Gansu, China \\ 3 School of Information Systems and Technology, \\ University of Wollongong, NSW, Australia \\ jshen@uow.edu.au,f1626@uowmail.edu.au \\ 4 Department of EE and CS, Research Lab of Electronics, \\ Massachusetts Institute of Technology, Cambridge, 02139, MA, USA
}

\begin{abstract}
General Vector Machine (GVM) is a newly proposed machine learning model, which is applicable to small samples forecast scenarios. In this paper, the GVM is applied into electricity load forecast based on single point modeling method. Meanwhile, traditional time series forecast models, including back propagation neural network (BPNN), Support Vector Machine (SVM) and Autoregressive Integrated Moving Average Model (ARIMA), are also experimented for single point electricity load forecast. Further, the combined model based on GVM, BPNN, SVM and ARIMA are proposed and verified. Results show that GVM performs better than these traditional models, and the combined model outperforms any other single models for single point electricity load forecast.
\end{abstract}

Keywords: General Vector Machine, Electricity load forecast, Time series forecast, Combined model

\section{Introduction}

Electricity load forecast is the base of electrical power system planning, which plays an important role in insurance of national life and stability of social economy. Hence, it has been a widely studied issue and many forecast methods are proposed. As a classical statistical learning model, Back propagation neural network (BPNN) is proved effective for electricity load [1]. However, limited to the finite training samples and surplus hidden nodes, BPNN often suffers from the over-fitting problem [2]. As shown in Fig. 1, the overall cost, which represents the error between actual values and forecasting values, usually has different trends

\footnotetext{
* Corresponding author: zhouqg@lzu.edu.cn
} 
in training samples and test samples. Generally, test cost will firstly reduce together with the training cost, and then it might increase after a minimum value. It means, BPNN model could possibly perform well on training samples, while it results in a poor performance for new situations.

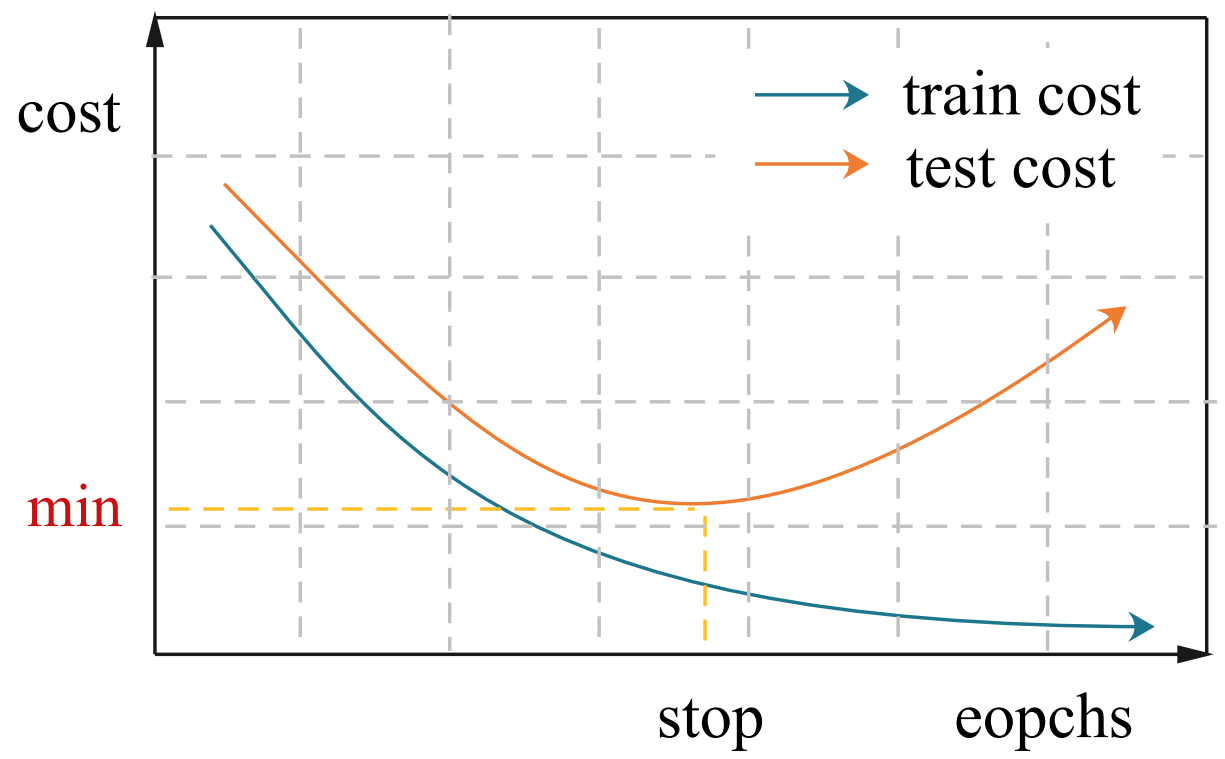

Fig. 1. The display of over fitting.

Due to the over-fitting problem, BPNN has issues in many different time series forecast scenarios. Meanwhile, over-fitting issue remains a controversial issue in the academic field. In order to deal with the problem, many approaches were proposed to eliminate the over-fitting phenomena [3].

Then, based on SLT, Vanpik proposed the famous Support Vector Machine (SVM) 4], which has solid theoretical foundation and trys to find a hyperplane that separates samples with a largest margin, is proved to be very effective for small samples. SVM evolves in a quicker pace than BPNN due to its solid theoretical foundation and excellent generalization ability for small samples [5]6. However, in the case when the training samples is vary small, SVM may select noise samples as support vectors, and hence misses the optimal model. Since Hiton proposed deep learning [7] in 2006, the neural network recovers once again. Arguments about the merits and demerits of neural network and SVM continues [8]. Meanwhile, some researchers try to find models that ensemble both the advantages of BPNN and SVM.

General Vector Machine (GVM) [10]11, which has a basic structure of threelayer neural network, is designed as a mixer model of neural network and SVM. In fact, GVM is applicable to cases of lacking samples [12[13], and it has been 
successfully applied in time series forecast problem, such as electricity demand forecast [14]. Meanwhile, development of modern technology generates the demand for more accurate electricity forecast. Therefore, many traditional models, e.g. BPNN [15, SVM [1617] are widely researched in electricity load forecast. In addition to the traditional models, some alternative approaches are also explored 1819. Unfortunately, due to the instability of electricity demand, electricity load forecast is still a great challenge. In this paper, we firstly applied GVM, BPNN, SVM and ARIMA into electricity load forecast. Then, the combined model of these four types models are researched.

The remainder of this paper is organized as follows. The introduction of GVM model is displayed in Section 2. Section 3 shows the single point forecast models, and applies it into electricity load forecast. The experiments and analysis are given in Section 4 . At last, Section 5 concludes our work.

\section{GVM model and the Monte Carlo training algorithm}

The GVM model and its training algorithm are shown in Fig. 2. Instead of support vectors, general vectors make the model not sensitive to individual sample, so as to gain the generalization ability of GVM model. In contrast to neural network, in the design of GVM, the parameter $\beta$ is imported into the hidden nodes to reduce the design risk. By adjusting $\beta$, GVM will keep robust for small fluctuations of the input feature vector.

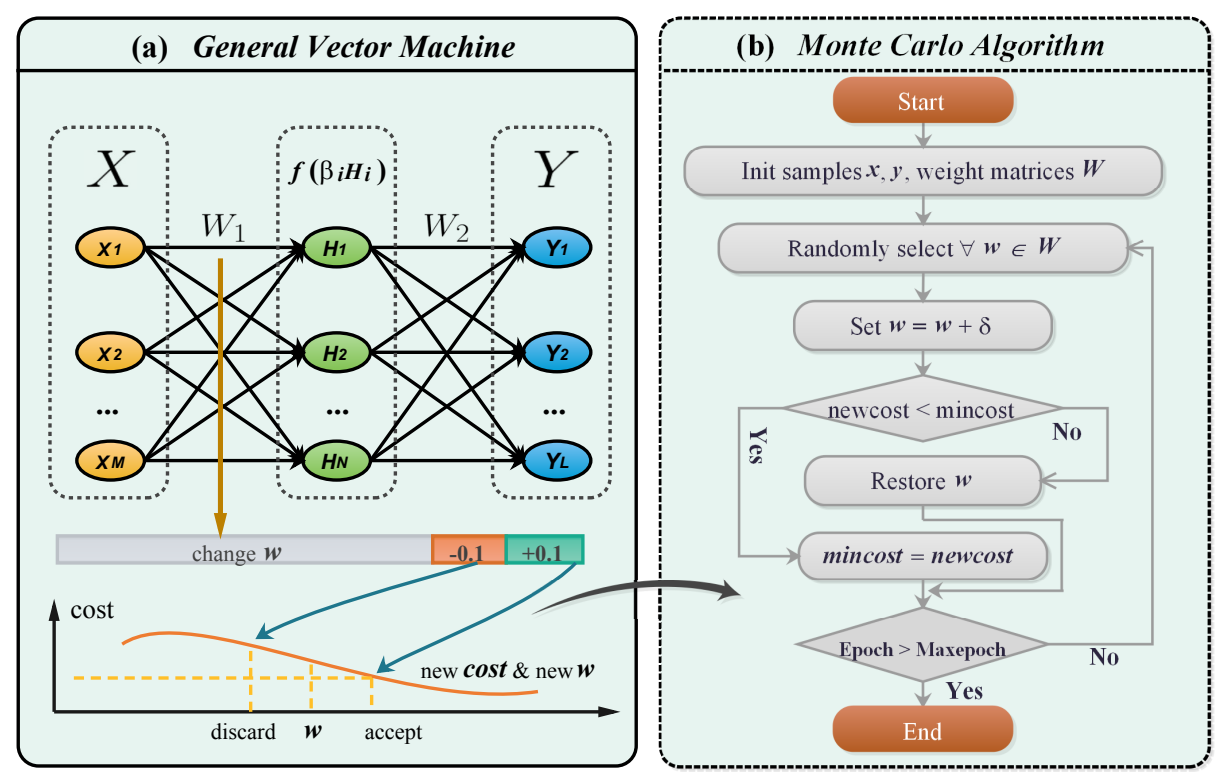

Fig. 2. (a) The structure of GVM. (b) The MC algorithm used to train GVM model. 
GVM introduces the Monte Carlo (MC) algorithm to train the model. The basic idea of training GVM is that the change of a weight would be accepted while the overall cost decreases. Specifically, it randomly changes one weight of the weight matrices in a small deviation, and GVM will accept the weight change while the overall cost is reduced. By this way, the training GVM model will gradually converge to a stable state. The pseudo-code of generating a new weight is given in Function (1), which repeatedly and randomly finds a new weight near the old one until the new weight is within the weight range.

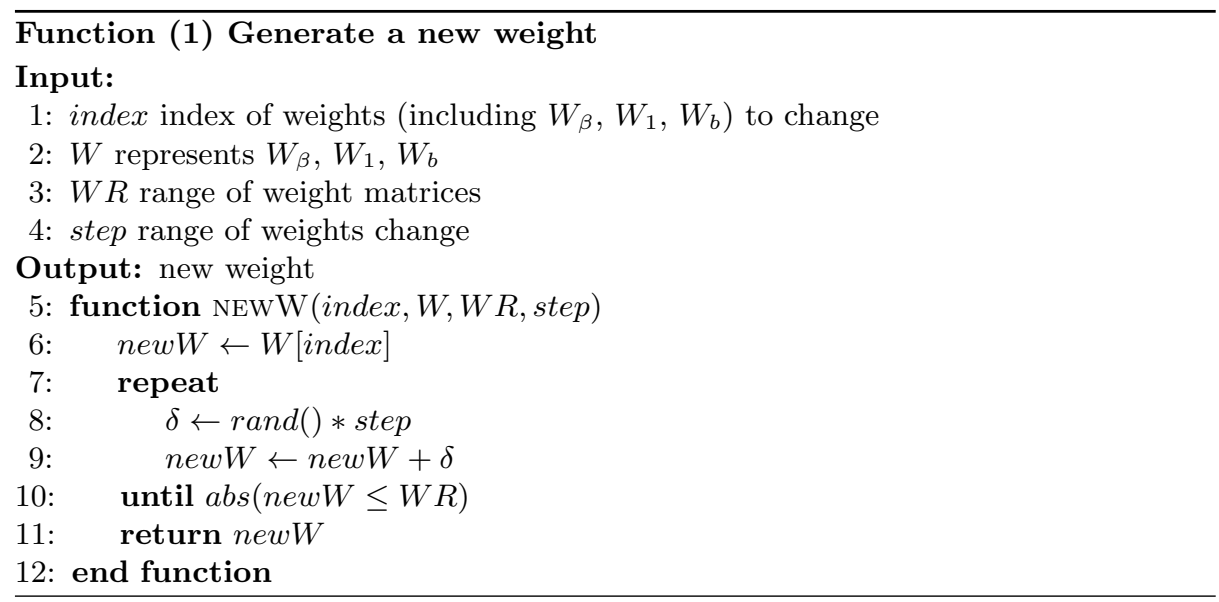

\section{Single point forecast modeling and the combined model}

In this paper, we focus on the forecast models for electricity load forecast. The electricity load data is collected from Queensland in Australia every half-hour, which means that there are 48 electricity load data per day. We select the data ranging from May 2, 2011 to July 3, 2011, which includes 3,024 data of 9 weeks. As shown in Fig. 3 a single point forecast model is constructed with 7 input nodes and 1 output node. The first 8 weeks data is used as the training data, in which the first 7 weeks data is used as the input vector and the eighth week data are seen as the output vector. When testing, the data from the second week to the ninth week is used as the test data, in which the data from the second week to the eighth week is used as input vector and the data of the ninth week is used as the output vector. The models to forecast the above input-output include GVM, BPNN, SVM and ARIMA. As talked above, BPNN represents a three-layer neural network, which is trained by back propagation algorithm. GVM is trained by MC algorithm. For SVM, time series forecast is in fact a regression problem (also called SVR). According to our experiments, the usage of radial basis function (RBF) as kernel function achieves better forecast results. 


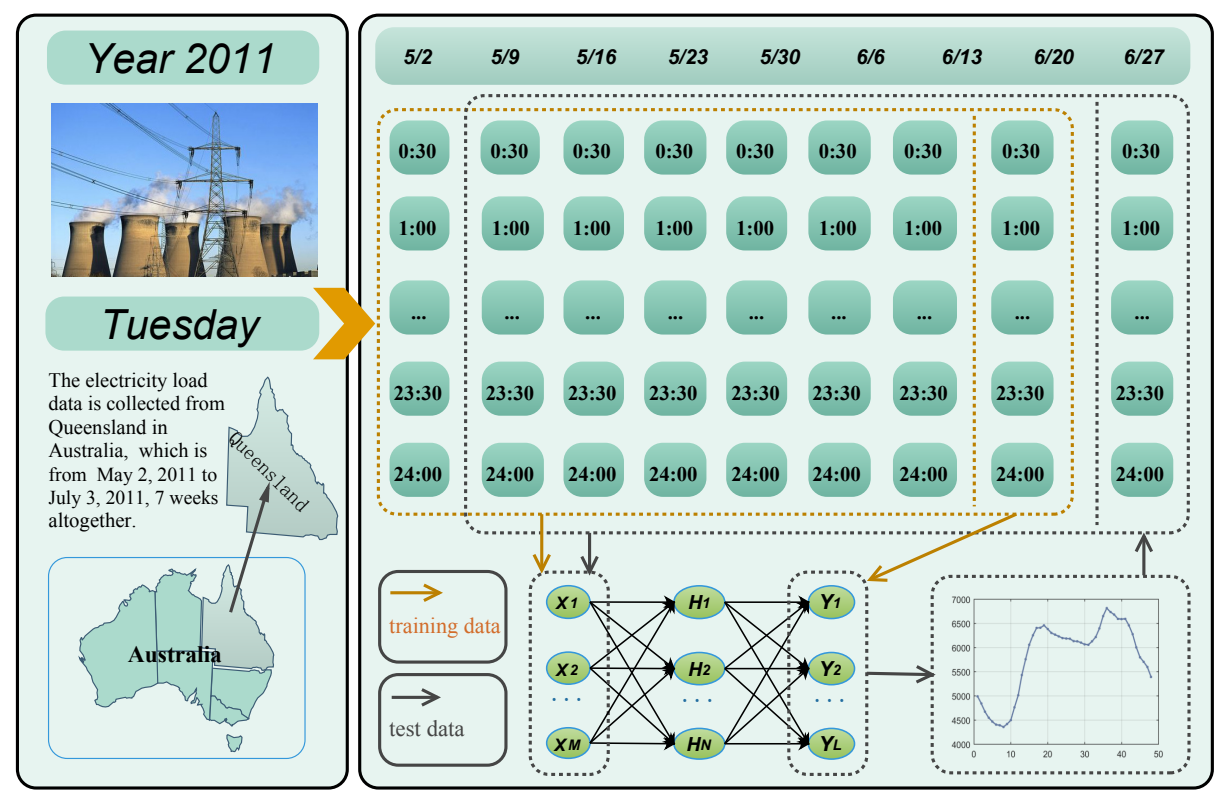

Fig. 3. Single point forecast modeling.

When selecting the parameters of regularization (penalty coefficient) $c$ and kernel width (radius of kernel function) $g$, the grid method is used to search the best regularization parameter $c$ and kernel parameter $g$.

In order to further improve the forecast performance, we try to combine the GVM, BPNN, SVM and ARIMA models. Similar to the training algorithm of GVM, the combined model is trained by Monte Carlo (MC) algorithm. That is, the weights of these four component models are initialized equally. Then, the weights are randomly changed in a small range, and the change of reducing the overall cost will be accepted.

\section{Experiments and analysis}

In this section, we will show the experiments results of single point forecast models and the combined model. Four metrics including Mean Absolute Percentage Error (MAPE), Mean Absolute Error (MAE) and Root Mean Square Error (RMSE) are used to evaluate the performances of these models.

\subsection{Comparisons on the single point forecast models}

For SVM model, we search the parameters $c$ and $g$ from $2^{-10}$ to $2^{10}$ divided by 0.1 to find the best pairs of $c$ and $g$, and the best parameters are listed in Table. 1. 
Table 1

The best regularization parameter $c\left(2^{x}\right)$ and the kernel parameter $g\left(2^{x}\right)$ of SVM model.

\begin{tabular}{lcccccccc}
\hline & Mon. & Tue. & Wed. & Thu. & Fri. & Sat. & Sun. & All \\
\hline$c\left(2^{x}\right)$ & 6.20 & 10.0 & 0.49 & 8.15 & 10.0 & 9.90 & -0.50 & -5.29 \\
$g\left(2^{x}\right)$ & -8.48 & -8.12 & -8.48 & -2.65 & -4.00 & -4.10 & -3.10 & -3.89 \\
\hline
\end{tabular}

The single point forecast results of four types models including GVM, GVM, BPNN, SVM and ARIMA are shown in Table 2, The best forecast results of single models are highlighted in boldfaces, which mainly occurs in GVM model. By comparing all the original models, we find that GVM performs the best in the models, which proves that GVM is suitable for electricity load forecast.

Table 2

Experimental results: the electricity load forecast results of single point models.

\begin{tabular}{ccccccccc}
\hline & \multicolumn{7}{c}{ MAPE $(\%)$} \\
\cline { 2 - 9 } & Mon. & Tue. & Wed. & Thu. & Fri. & Sat. & Sun. & All \\
\hline GVM & $\mathbf{0 . 9 8 0 3}$ & $\mathbf{1 . 4 5 9 1}$ & $\mathbf{1 . 7 6 9 2}$ & 3.1568 & 2.9631 & 2.2151 & 1.5976 & $\mathbf{2 . 3 6 1 4}$ \\
BPNN & 1.7990 & 1.8836 & 2.3726 & $\mathbf{3 . 0 6 7 6}$ & 3.2271 & 1.9492 & 1.5378 & 2.7536 \\
SVM & 3.4149 & 3.1351 & 3.1588 & 4.0683 & $\mathbf{1 . 4 1 6 0}$ & $\mathbf{1 . 5 2 1 9}$ & $\mathbf{1 . 0 5 3 6}$ & 3.3901 \\
ARIMA & 6.5651 & 4.6551 & 4.4776 & 6.4666 & 5.2850 & 1.8155 & 1.1165 & 4.3696 \\
\hline & & & & MAE & & & & \\
& Mon. & Tue. & Wed. & Thu. & Fri. & Sat. & Sun. & All \\
\hline GVM & $\mathbf{5 8 . 6 1 7}$ & $\mathbf{8 6 . 9 9 0}$ & $\mathbf{1 0 5 . 0 3}$ & $\mathbf{1 7 7 . 6 2}$ & 170.15 & 115.07 & 83.592 & $\mathbf{1 3 1 . 1 3}$ \\
BPNN & 104.62 & 110.32 & 141.53 & 182.69 & 193.01 & 106.62 & 82.538 & 155.23 \\
SVM & 196.06 & 189.32 & 172.14 & 230.15 & $\mathbf{8 3 . 8 9 0}$ & $\mathbf{7 8 . 6 6 5}$ & 82.538 & 179.49 \\
ARIMA & 382.26 & 269.71 & 267.67 & 377.4 & 300.38 & 94.516 & $\mathbf{5 7 . 5 3 0}$ & 249.66 \\
\hline & & & & RMSE & & & & \\
\hline & Mon. & Tue. & Wed. & Thu. & Fri. & Sat. & Sun. & All \\
\hline GVM & $\mathbf{8 2 . 8 0 9}$ & $\mathbf{1 1 0 . 8 6}$ & $\mathbf{1 5 5 . 5 2}$ & $\mathbf{1 9 8 . 6 6}$ & 185.57 & 136.68 & 96.127 & $\mathbf{1 5 6 . 6 1}$ \\
BPNN & 134.78 & 132.80 & 168.90 & 202.21 & 219.74 & 128.90 & 104.07 & 193.13 \\
SVM & 237.57 & 214.14 & 214.23 & 301.05 & $\mathbf{1 2 4 . 4 8}$ & $\mathbf{9 2 . 7 0 1}$ & 104.07 & 214.28 \\
ARIMA & 495.86 & 339.90 & 328.75 & 445.09 & 341.68 & 110.81 & $\mathbf{7 2 . 0 9 0}$ & 301.13 \\
\hline
\end{tabular}

The boldfaces are best results. 


\subsection{Comparisons on the combined model}

In this subsection, the combined model ensembles GVM, BPNN, SVM and ARIMA are tested. As discussed above, MC algorithm is adopted to find the combined weights of component models. After a period of training, the combined weights almost keep stable, which are shown in Table 3. We can see that the combined models are able to take full use of the advantages of each component model, and achieve higher forecasting precisions than component models. The forecast results of the combined model (red curve) and the actual (blue curve) are shown in Fig. 4. We can see that the electricity load is well forecast.

Table 3

Experimental results: the combined weights of component models, and MAPE, MAE, and RMSE of the combined model.

\begin{tabular}{llllllll}
\hline & $\operatorname{GVM}(\omega 1)$ & $\operatorname{BPNN}(\omega 2)$ & $\operatorname{SVM}(\omega 3)$ & ARIMA $(\omega 4)$ & MAPE & MAE & RMSE \\
\hline Mon. & 0.5491 & 0.3953 & 0.0329 & 0.0226 & 0.92712 & 51.392 & 61.277 \\
Tue. & 0.5692 & 0.4255 & -0.0079 & 0.0132 & 1.1288 & 65.577 & 78.902 \\
Wed. & 0.3635 & 0.5963 & -0.0343 & 0.0745 & 1.3029 & 77.097 & 113.24 \\
Thu. & 0.2900 & 0.6502 & 0.0303 & 0.0295 & 1.3792 & 76.851 & 101.47 \\
Fri. & 0.2317 & 0.25223 & 0.2883 & 0.2277 & 1.3591 & 74.714 & 100.07 \\
Sat. & -0.0848 & 0.2250 & 0.7316 & 0.1283 & 1.4975 & 78.753 & 88.717 \\
Sun. & 0.0444 & 0.0209 & 0.7470 & 0.1877 & 1.0346 & 53.34 & 61.441 \\
All & 1.6407 & 0.1281 & -0.5353 & -0.2335 & 1.7143 & 98.975 & 128.81 \\
\hline
\end{tabular}

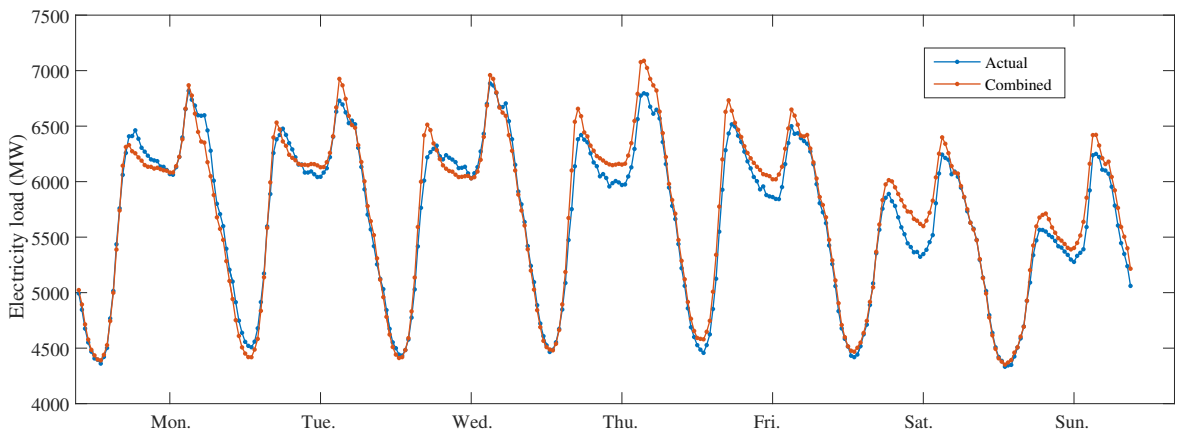

Fig. 4. Experimental results: the electricity load forecast results of combined model. 


\section{Conclusion}

Electricity load forecast, as an important and difficult time series forecast issue, has been researched by single point modeling method in this paper. As a newly proposed model, GVM has also been applied into electricity load forecast and achieved better forecast results than other traditional forecast models. Further, the combined model ensembles GVM and traditional BP, SVM and ARIMA are explored in this paper. Meanwhile, MC algorithm is pioneered to find the combined weights of the component models. Results demonstrate that the combined models outperforms the component models and $\mathrm{MC}$ is effective for training combined model.

\section{Acknowledgments}

This work was partially supported by National Natural Science Foundation of China under Grant No. 61402210, The Fundamental Research Funds for the Central Universities under Grant No. lzujbky-2018-k12, Ministry of Education - China Mobile Research Foundation under Grant No. MCM20170206, Major National Project of High Resolution Earth Observation System under Grant No. 30-Y20A34-9010-15/17, State Grid Corporation Science and Technology Project under Grant No. SGGSKY00FJJS1700302, No. 52272218002K and No. SGGSKY00FJJS1800403, Program for New Century Excellent Talents in University under Grant No. NCET-12-0250, and Strategic Priority Research Program of the Chinese Academy of Sciences with Grant No. XDA03030100.

\section{References}

1. A. Laouafi, M. Mordjaoui, D. Dib, One-hour ahead electric load and wind-solar power generation forecasting using artificial neural network, Renewable Energy Congress (2015) 1-6.

2. D. M. Hawkins, The problem of overfitting, Cheminform 35 (19) (2004) 1.

3. Y. Gao, X. Kong, C. Hu, Z. Zhang, H. Li, L. Hou, Multivariate data modeling using modified kernel partial least squares, Chemical Engineering Research \& Design 94 (2015) 466-474.

4. C. Cortes, V. Vapnik, Support-Vector Networks, Kluwer Academic Publishers, 1995.

5. C. Sch, I. Laptev, B. Caputo, Recognizing human actions: A local svm approach, International Conference on Pattern Recognition 3 (2004) 32-36.

6. L. J. Cao, F. E. H. Tay, Support vector machine with adaptive parameters in financial time series forecasting, IEEE Transactions on Neural Networks 14 (6) (2004) 1506-1518.

7. G. E. Hinton, S. Osindero, Y. W. Teh, A fast learning algorithm for deep belief nets, MIT Press, 2006.

8. J. Ren, Ann vs. svm: Which one performs better in classification of mccs in mammogram imaging, Knowledge-Based Systems 26 (2012) 144-153. 
9. A. S. Ahmad, M. Y. Hassan, M. P. Abdullah, H. A. Rahman, F. Hussin, H. Abdullah, R. Saidur, A review on applications of ann and svm for building electrical energy consumption forecasting, Renewable \& Sustainable Energy Reviews 33 (2) (2014) 102-109.

10. H. Zhao, General vector machine, arXiv preprint.

11. B. Yong, F. Li, Q. Lv, J. Shen, Q. Zhou, Derivative-based acceleration of general vector machine, Soft Computing 23 (3) (2019) 987-995.

12. B. Yong, Z. Xu, J. Shen, H. Chen, Y. Tian, Q. Zhou, Neural network model with monte carlo algorithm for electricity demand forecasting in queensland 47 (2017) $1-7$.

13. B. Yong, J. Shen, Z. Shen, H. Chen, X. Wang, Q. Zhou, Gvm based intuitive simulation web application for collision detection, Neurocomputing 279 (2) (2017) 63-73.

14. B. Yong, L. Huang, F. Li, J. Shen, X. Wang, Q. Zhou, A research of monte carlo optimized neural network for electricity load forecast, The Journal of Supercomputing (2019) 1-14.

15. C. Yang, G. Deconinck, W. Gui, An optimal power-dispatching control system for the electrochemical process of zinc based on backpropagation and hopfield neural networks, IEEE Transactions on Industrial Electronics 50 (5) (2003) 953-961.

16. M. V. Suganyadevi, C. K. Babulal, Support vector regression model for the prediction of loadability margin of a power system, Applied Soft Computing Journal 24 (2014) 304-315.

17. A. Selakov, S. Mellon, D. Bekut, Hybrid pso-svm method for short-term load forecasting during periods with significant temperature variations in city of burbank, Applied Soft Computing 16 (3) (2014) 80-88.

18. G. Zahedi, S. Azizi, A. Bahadori, A. Elkamel, S. R. W. Alwi, Electricity demand estimation using an adaptive neuro-fuzzy network: A case study from the ontario province canada, Energy 49 (1) (2013) 323-328.

19. P. Morris, D. Vine, L. Buys, Application of a bayesian network complex system model to asuccessful community electricity demand reduction program, Energy 84 (2015) 63-74. 